Journal of Bangladesh Academy of Sciences, Vol. 36, No. 1, 97-107, 2012

\title{
OPTICAL AND TRANSPORT PROPERTIES OF p-TYPE GaAs
}

\author{
MEHNAZ SHARMIN, SHAMIMA CHOUDHURY*, NASRIN AKHTAR ${ }^{1}$ AND \\ TAHMINA BEGUM ${ }^{1}$
}

Department of Physics, University of Dhaka, Dhaka-1000, Bangladesh

\begin{abstract}
Electrical properties such as electrical resistivity, Hall coefficient, Hall mobility, carrier concentration of p-type GaAs samples were studied at room temperature (300 K). Resistivity was found to be of the order of $5.6 \times 10^{-3} \Omega-\mathrm{cm}$. The Hall coefficient $\left(\mathrm{R}_{\mathrm{H}}\right)$ was calculated to be $7.69 \times$ $10^{-1} \mathrm{~cm}^{3} / \mathrm{C}$ and Hall mobility $\left(\mu_{\mathrm{H}}\right)$ was found to be $131 \mathrm{~cm}^{2} / \mathrm{V}$-s at room temperature from Hall effect measurements. Carrier concentration was estimated to be $8.12 \times 10^{18} / \mathrm{cm}^{3}$ and the Fermi level was calculated directly from carrier density data which was $0.33 \mathrm{eV}$. Photoconductivity measurements were carried on by varying sample current, light intensity and temperature at constant chopping frequency $45.60 \mathrm{~Hz}$ in all the cases mentioned above. It was observed that within the range of sample current $0.1-0.25 \mathrm{~mA}$ photoconductivity remains almost constant at room temperature $300 \mathrm{~K}$ and it was found to be varying non-linearly with light intensity within the range 37 - 12780 lux. Photoconductivity was observed to be increasing linearly with temperature between 308 and $428 \mathrm{~K}$. Absorption coefficient $(\alpha)$ of the samples has been studied with variation of wavelength (300 $2500 \mathrm{~nm}$ ). The value of optical band gap energy was calculated between 1.34 and $1.41 \mathrm{eV}$ for the material from the graph of $(\alpha \mathrm{h} v)^{2}$ plotted against photon energy. The value of lattice parameter (a) was found to be $5.651 \AA$ A by implying X-ray diffraction method (XRD).
\end{abstract}

Key words: Photoconductivity, Band gap energy, Absorption coefficient, Lattice parameter

\section{INTRODUCTION}

GaAs is a III-V compound semiconductor with large band gap. This is a direct band gap material, very useful material in the field of electronics and optoelectronics. It is a very promising material which is used in construction of solid state detectors, high efficiency solar cells, Infrared LEDs, Lasers diodes, Gunn diodes, radar systems etc.

The hole carrier concentrations of $\mathrm{Zn}$ doped GaAs was studied by using the Hall effect (Hudait et al. 1997). The hole concentration and mobility in wafers from (001) monocrystal GaAs doped with $\mathrm{Zn}$ was measured by the Hall effect method (Mihailova 2005) under constant electrical and magnetic fields. The absorption coefficient of

*Corresponding author: <skc.phy@gmail.com>.

${ }^{1}$ Experimental Physics Division, Atomic Energy Centre, Dhaka-1000, Bangladesh. 
Gallium Arsenide varying thickness (0.6 $\mu \mathrm{m}-6 \mathrm{~mm})$ was measured (Sturge 1962) over the range of photon energy 0.6 to $2.75 \mathrm{eV}$ at temperature from 10 to $294 \mathrm{~K}$. According to the worker the band gap energy was deduced to be $1.435 \mathrm{eV}$ at $294 \mathrm{~K}$. The optical absorption coefficient of $\mathrm{Zn}$ doped GaAs with doping concentration $7.37 \times 10^{16} \mathrm{~cm}^{-3}$ was also investigated (El-Nahass et al. 2011) over the wavelength range $930-2500 \mathrm{~nm}$ and the value of direct energy band gap was found to be $1.38 \mathrm{eV}$ at room temperature condition. Photoconductivity techniques were applied to study various transport parameters of $\mathrm{Cr}$ doped p-type GaAs crystal (Longeaud et al. 2006). Optical and transport properties of n-type GaAs were investigated by Chowdhury et al. (2010).

Since, GaAs is a direct band gap semiconductor, recombination occurs in GaAs without a change in momentum and most of the energy appears as photon energy with a wavelength dependent on the band gap energy. Energy band gap is an important property of a semiconductor that decides to what extent a semiconductor is useful in fabrication of optoelectronic devices like lasers, LEDs etc.

Photoconductivity is a phenomenon that is exhibited by semiconductors under suitable experimental conditions. This occurs when an incident light beam impinges upon a semiconductor and causes an increase in electrical conductivity. Photoconductive response of a semiconductor also gives an idea, to what extent the material is suitable to be used in optoelectronic devices. The materials which give nice photoconductive response are very useful in construction of infrared solid state detectors.

Present study was carried on by measuring the electrical, optical and structural properties of heavily Zn doped GaAs. Electrical parameters of p-type GaAs such as resistivity, carrier concentration, Hall coefficient, Hall mobility and Fermi level were determined.

Optical process or absorption method was implied to determine the band gap energy of p-type GaAs samples. Transmission and reflection coefficients of those samples were measured at wavelengths ranging from $300-2500 \mathrm{~nm}$. The absorption coefficients were calculated and energy band gap of p-type GaAs samples was determined.

Photoconductive response of the p-type GaAs samples were also investigated under various experimental conditions such as by varying sample current, light intensity and temperature. In this paper, the modulated illumination technique has been implemented for measuring steady state photoconductivity.

\section{MATERIALS AND METHOD}

Rectangular shaped pieces of different dimensions were cut by a diamond cutter from a p-type single crystal GaAs wafer of diameter $5 \mathrm{~cm}$ and of thickness $0.029 \mathrm{~cm}$ and four samples of different dimensions (length varying between 0.93 and $1.6 \mathrm{~cm}$ and 
breadth varying between 0.58 and $0.61 \mathrm{~cm}$ ) were prepared. The mechanically damaged surfaces of the samples were polished with 100 grit $\mathrm{SiC}$ powder and 1.0 micron alpha alumina powder, respectively in polisher (Buehler Ecomet III Grinder, U.S.A). The samples were then cleaned with acetone and de-ionized water in an ultrasonic bath (Max Knol 1959). Electrical leads were soldered to the end faces of the samples by Indium.

Electrical resistivity of the p-type GaAs samples were measured by potential probe method at room temperature. Hall coefficient was measured by four probe d.c. compensation method. The experimental circuit arrangement was described in (Akhtar and Tahmina 2005). A water cooled electromagnet (N100, New port series, UK) with pole pieces $10 \mathrm{~cm}$ in diameter and capable of producing magnetic field (1.96 - 5.41 Gauss) with a gap of $2 \mathrm{~cm}$ between the pole tip faces was used for Hall Effect measurement.

Modulated illumination method was implemented for measuring photoconductivity using a DSP lock-in-amplifier (7225, Signal Recovery). Details concerning optical experimental set up was described (Akhtar et al. 1999). A $100 \mathrm{~W}$ quartz halogen lamp was used as light source. A light chopper (125/99, EG and G, PARC) to measure chopping frequency and lenses were used for optical measurements. A system of glass lenses ensured uniform illumination of the sample surfaces. Intensity of light was measured by a lux meter (LX 101).The lamp was first calibrated to ensure the desired intensity with the help of a lux meter. Temperature of the sample was increased above room temperature with the help of a heating coil ( $35 \mathrm{~W})$ and power supply (Philips).The temperature was measured using $\mathrm{Cu}$-Constantan thermocouple and digital temperature controller. Heating rate was maintained to be $0.00533 \mathrm{~K} / \mathrm{sec}$.

The optical transmission and reflection coefficients of the samples were observed with the variation of wavelength of light, ranging from $300-2500 \mathrm{~nm}$ incident on the surfaces of the samples. A dual beam UV-VIS-NIR recording spectrophotometer (Shimadzu, UV-3100) was used for this purpose. Light source of the units was $50 \mathrm{~W}$ Tungsten-Halogen lamp and Deuterium lamp. An integrating sphere detected signal was coming from the samples.

Lattice parameter of the sample was determined by X-ray diffraction (XRD) method using a Philips PW 3040 X' Pert PRO XRD system with $\mathrm{Cu}-\mathrm{K} \alpha$ radiation, operated at 40 $\mathrm{kV}$ and $30 \mathrm{~mA}$, with angular range $20^{\circ} \leq 2 \theta \leq 80^{\circ}$.

\section{THEORITICAL FORMULATION}

Hall coefficient $\left(\mathrm{R}_{\mathrm{H}}\right)$ was determined using the following equation,

$$
R_{H}=\frac{t V_{H}}{H I} \times 10^{8}
$$


Where $t$ is the sample thickness in $\mathrm{cm}, \mathrm{V}_{\mathrm{H}}$ is the Hall voltage in volt, $\mathrm{H}$ is the magnetic field in Gauss and $\mathrm{I}$ is the current in A.

Carrier density was computed from Hall data using the relation as follows,

$$
p=\frac{1}{e R_{H}}
$$

Where $e$ is the charge of the electron and $R_{H}$ is the Hall coefficient. Data for conductivity $(\sigma)$ and Hall coefficient $\left(R_{H}\right)$ were combined to give Hall mobility $\mu_{H}$ defined by the relation,

$$
\mu_{H}=\sigma R_{H}
$$

The carrier density data is given (Brehm and Pearson 1972) by the following equation,

$$
p=2\left(\frac{m^{*}{ }_{p} K T}{2 \pi h^{2}}\right)^{\frac{3}{2}} \exp \left(-\frac{E_{F}}{K T}\right)
$$

Where $\mathrm{m}_{\mathrm{p}}{ }^{*}=0.50 \mathrm{~m}_{\mathrm{e}}$ is used for $\mathrm{p}$-type GaAs samples and $\mathrm{m}_{\mathrm{e}}$ is the mass of an electron.

From equation (4), the Fermi level is described by,

$$
E_{F}=K T\left(\ln 2-\ln p+\frac{3}{2} \ln \left(\frac{m_{p}^{*} K T}{2 \pi h^{2}}\right)\right.
$$

The transmission coefficient $\mathrm{T}$ of a thin semiconductor sample is given by Schroder, (1998)

$$
T=\frac{(1-R)^{2} \exp (-\alpha d)}{\left(1-R^{2}\right) \exp (-2 \alpha d)}
$$

Where $\mathrm{T}$ and $\mathrm{R}$ are the transmission and reflection coefficients and $\mathrm{d}$ is the thickness of the sample.

From the first order approximation of exponential terms of Eq. (6), the absorption coefficient $\alpha$ is derived as,

$$
\alpha=\frac{1}{d}\left[1-\frac{\left\{T\left(R^{2}+1\right)\right\}}{\left\{(R-1)^{2}+2 T R^{2}\right\}}\right]
$$

Energy band $\mathrm{E}_{\mathrm{g}}$ of materials is related to absorption coefficient $\alpha$ (Joshi et al. 2002)

$$
\alpha h v=A\left(h v-E_{g}\right)^{n}
$$

Where $\mathrm{A}$ is a constant, hv the photon energy, $\mathrm{E}_{\mathrm{g}}$ is the band gap and $\mathrm{n}$ is an index which assumes that the values 1/2, 3/2, 2 and 3 depending on the nature of the electronic transition responsible for the absorption, $n=1 / 2$ is taken for an allowed direct transition . 
The theory for photoconductivity measurement with lock-in-amplifier is shown (Ryvkin 1964). The change in conductance when sample being illuminated is given (Ryvkin 1964) by the following relation

$$
\Delta \sigma=\frac{\Delta V\left(R+r_{0}\right)}{r_{0}^{2} V R-\Delta V r_{0} R\left(R+r_{0}\right)}
$$

Where $\Delta V$ the change in voltage when the sample is illuminated, $R$ is the load resistance, $r_{\mathrm{o}}$ is the dark resistance of the sample and $\mathrm{V}$ is the supply voltage.

When $R=r_{\mathrm{o}}$ (Maximum sensitive regime), $\Delta V$ attains the maximum value $\Delta V_{m}$ and photoconductivity $\Delta \sigma_{p c}^{*}$ can be expressed in terms of dark conductivity $\left(\sigma_{0}\right)$ of the sample.

$$
\Delta \sigma_{p c}^{*}=4 \cdot \frac{\Delta V_{m}}{V} \cdot \sigma^{0}
$$

The optimum value of $\Delta V_{m}$ is measured with a lock in amplifier in which voltage developed across $R$ is fed to one input channel and the output of the photocell produced by chopped light is used as reference frequency.

\section{RESULTS AND DISCUSSIONS}

Potential probe method was implied for measuring the resistivity of p-type GaAs samples. The current-voltage (I-V) characteristics of the three samples are shown in Fig.1. The linearity of the graphs indicated that electrical contacts to the samples were ohmic. The resistivity of the samples was found to be of the order $5.67 \times 10^{-3} \Omega$-cm from the I-V data.

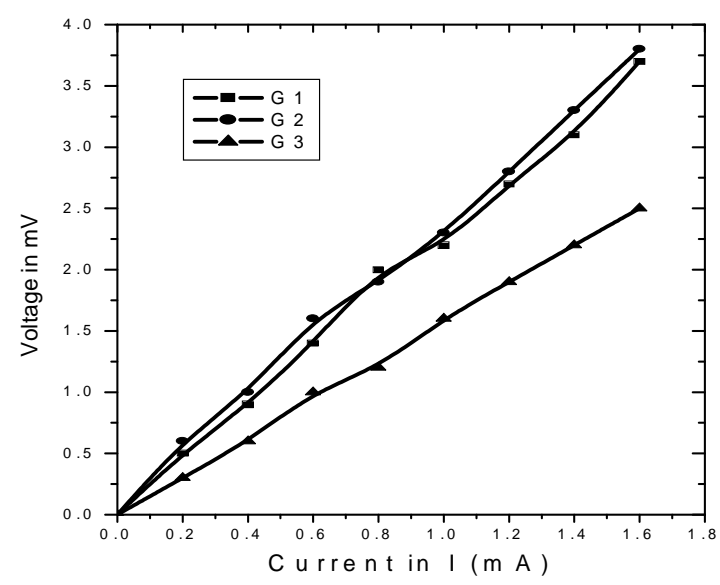

Fig. 1. Current versus voltage (I - V) curves for p-GaAs samples. 


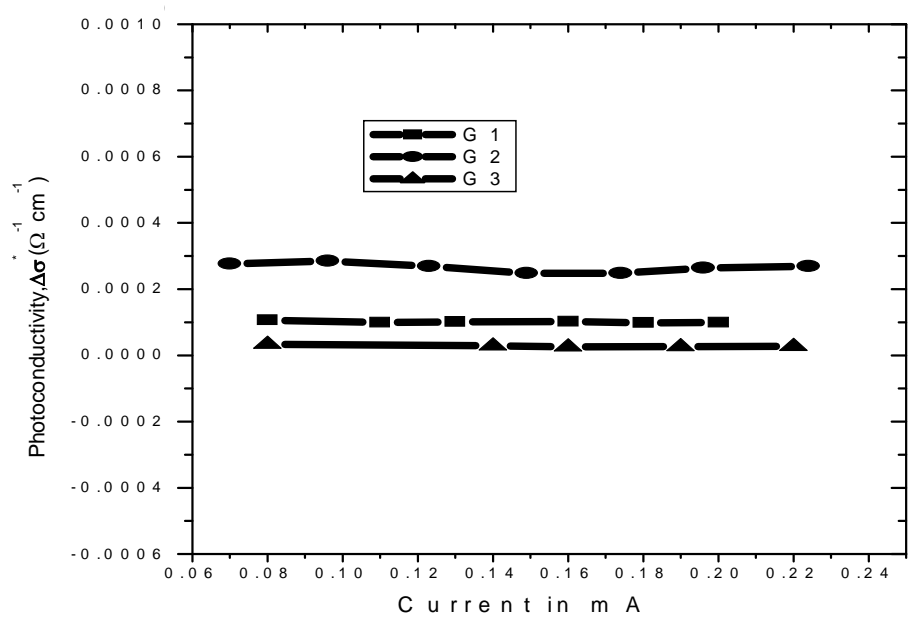

Fig. 2. Photoconductivity versus current curves for p-type GaAs samples.

Photoconductivity of p-type GaAs was calculated by the Eq. (10). The dark conductivity $\left(\sigma^{0}\right)$ of all the samples lies between $30-69 \Omega^{-1} / \mathrm{cm}$. Photoconductivity of the samples falls within $2.53 \times 10^{-5}(\Omega-\mathrm{cm})^{-1}$ to $2.69 \times 10^{-5}(\Omega-\mathrm{cm})^{-1}$. The Fig. 2 shows photoconductivity as a function of sample current; from the figure this is clear that photoconductivity remains almost constant with the variation of sample current.

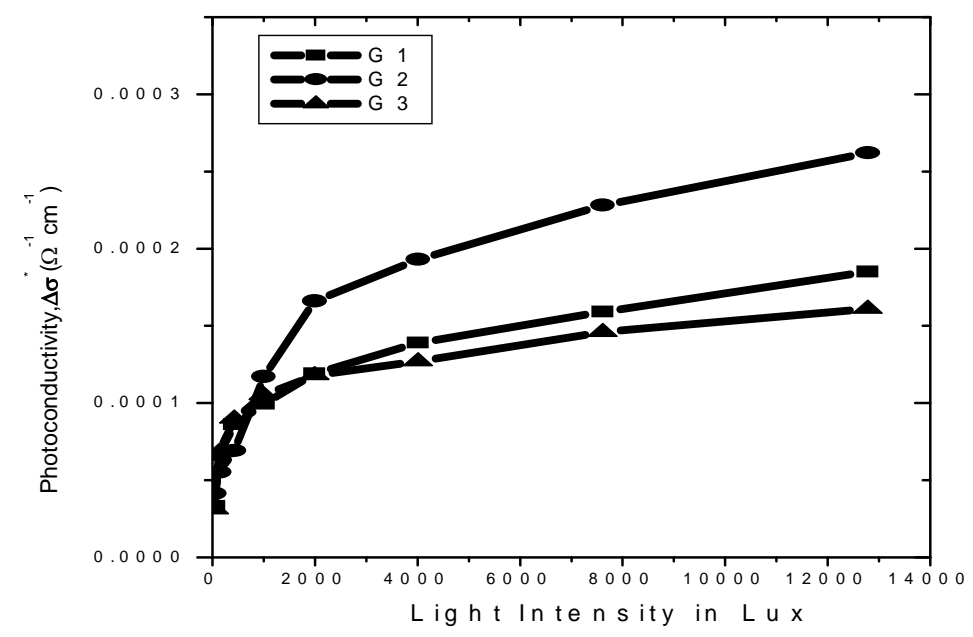

Fig. 3. Photoconductivity as a function of light intensity.

Fig.3 shows Photoconductivity $\left(\Delta \sigma^{*}{ }_{p c}\right)$ as a function of light intensity $\left(\mathrm{I}_{\mathrm{L}}\right)$, which indicates the increase of photoconductivity within the range $37-12780$ Lux. The light intensity was controlled by varying the voltage of the light source. The sample was 
illuminated over its entire surface. Since, increase of light intensity causes increase of excess minority carriers; photoconductivity is increased as a result of it.

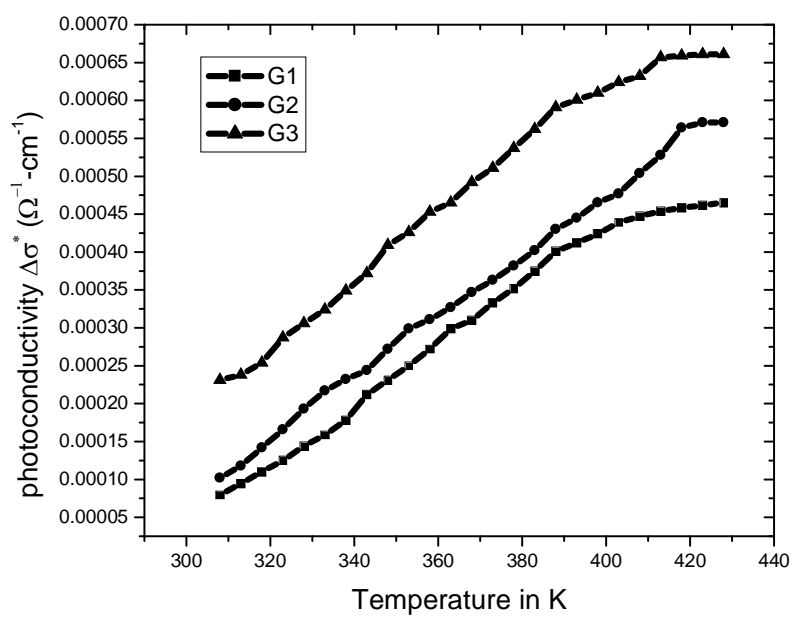

Fig . 4. Photoconductivity as a function of temperature.

Photoconductivity was also studied with the variation of temperature region 308 $428 \mathrm{~K}$. It is found that photoconductivity increases with the increase of temperature. This occurs due to the increase of minority carrier concentration with temperature. The nature of the graph as shown in Fig. 4 was almost linear in the range of temperature mentioned above.

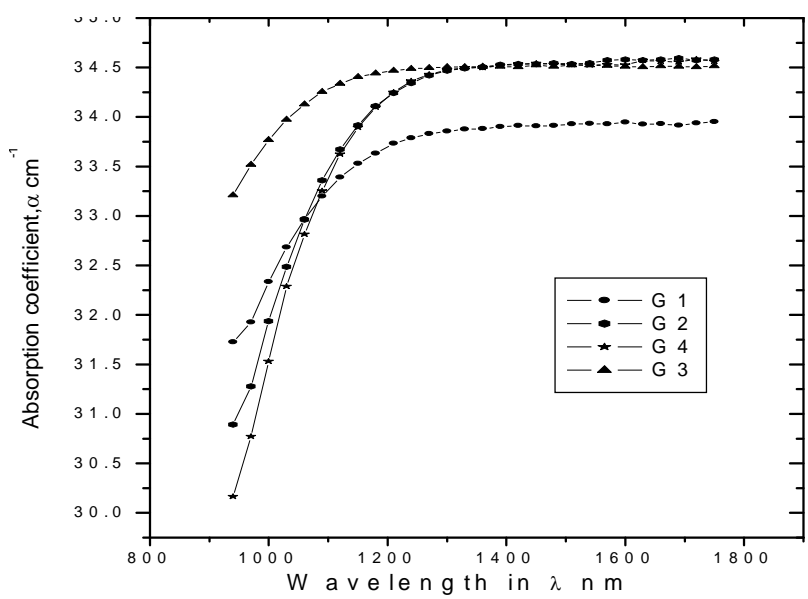

Fig. 5. Absorption coefficient as a function of photon wavelength $(\lambda)$.

The absorption coefficient $(\alpha)$ of mechanically polished p-type GaAs was computed from the measurement of transmission and reflection coefficients at different photon 
wavelengths ranging from $300-2500 \mathrm{~nm}$. Absorption coefficient $(\alpha)$ is plotted against photon wavelength $(\lambda)$ as shown in Fig. 5 for p-type GaAs.

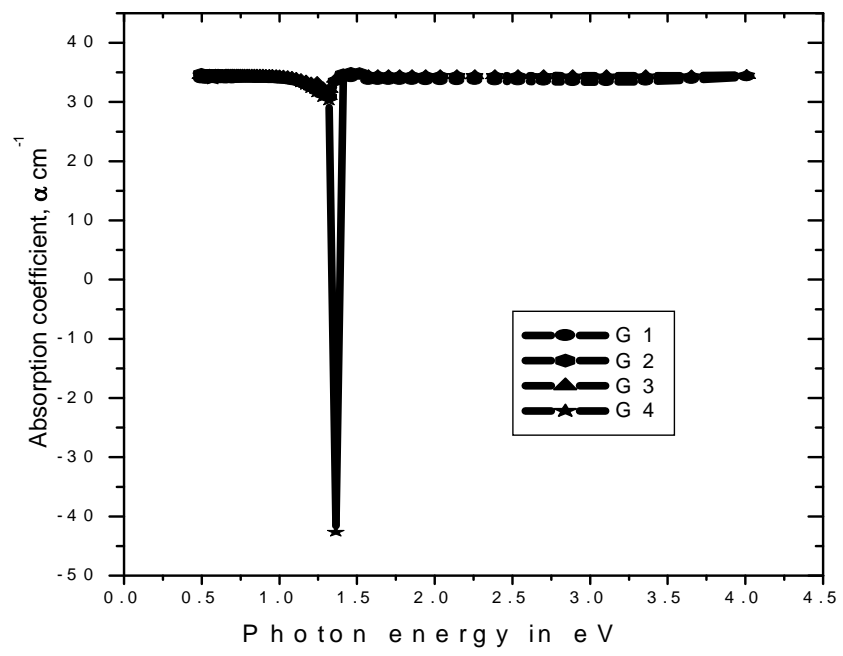

Fig. 6. Absorption coefficient as a function of photon energy (E).

Fig. 6 shows the variation of absorption coefficient $(\alpha)$ with photon energy (E). It is ascertained that the curve has a distinct peak at $1.40 \mathrm{eV}$ and the beginning of a strong absorption band setting in at $0.89 \mathrm{eV}$.

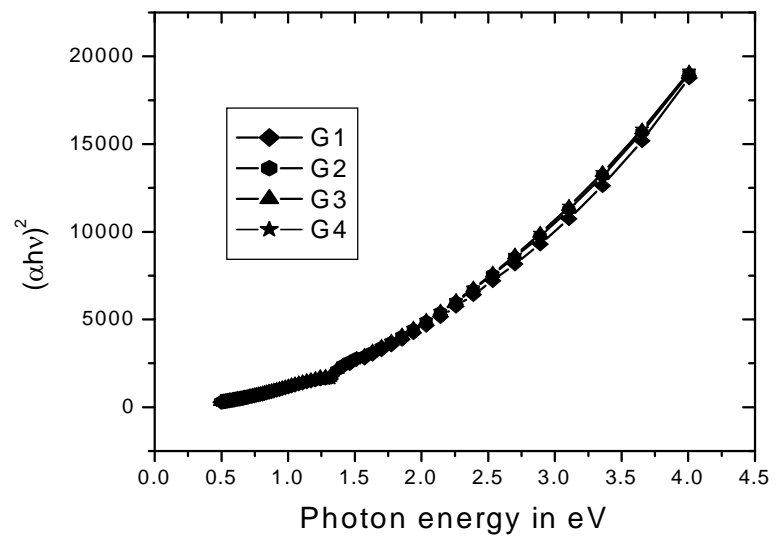

Fig. 7. $(\alpha h v)^{2}$ versus photon energy curves.

Fig. 7 shows the plot of $(\alpha h v)^{2}$ as a function of photon energy hv which was used to calculate the band gap energy of p-type GaAs. The straight portion of the curves indicated direct transition. The extrapolation of the straight line to $[(\alpha h v)]^{2}=0$ gave the 
value of direct band gap 1.41, 1.375, 1.38 and $1.34 \mathrm{eV}$ for the four samples of p-type GaAs.

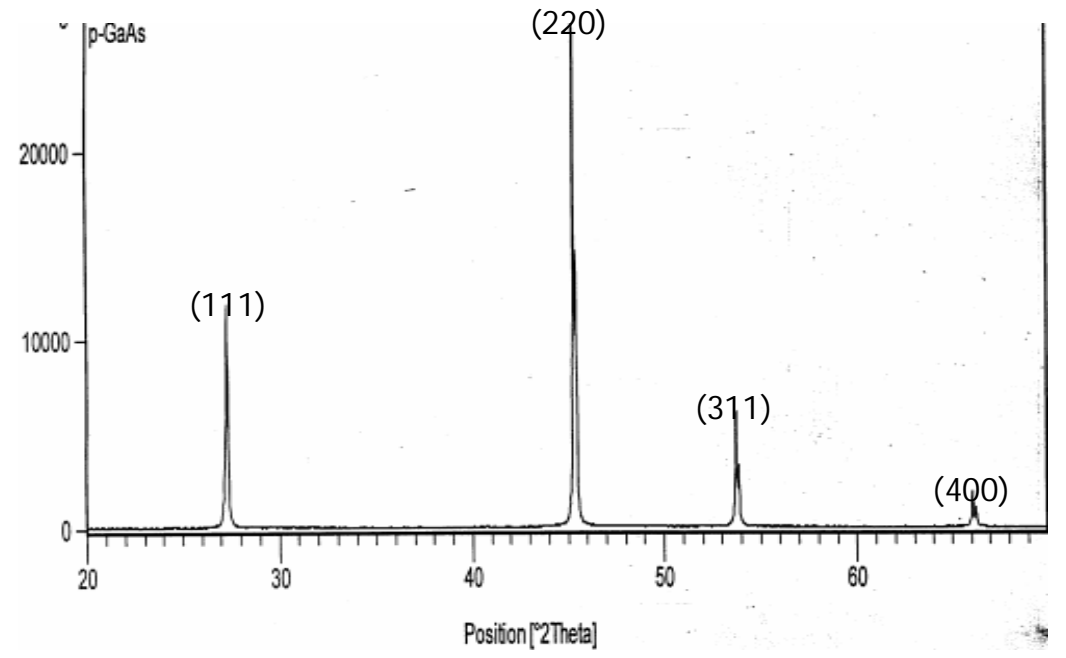

Fig. 8. The X-ray diffraction pattern of p-type GaAs.

The X-ray diffraction (XRD) pattern of p-type GaAs is shown in Fig.8 which was consistent with the XRD pattern of cubic lattice structure ( $\mathrm{ZnS}$ structure) and similar to that found by other workers in the case of GaAs (Baublitz 1982, Sadwick 1996) Lattice parameter is calculated to be $5.651 \AA$ from the XRD data which was near the standard value $5.654 \AA$ (Madelung 1964). Some impurity peaks were found in the diffraction pattern, which may be due to p-type doping.

From the absorption coefficient measurements, it was observed that absorption coefficient increases parabolically with the wavelength above the fundamental absorption edge of the curve (Fig. 5). It is consistent with the analysis to give the direct band gap nature (Omar 2003). The fundamental edge usually occurs in the infrared region because the band gap of the semiconductors is small- frequently $1 \mathrm{eV}$ or less (Omar 2003). From $0.5-1.2 \mathrm{eV}$ the absorption decreases, this may be due to the presence of some unknown impurity and its ionization effect. In the region around $1.2 \mathrm{eV}$ excitation across the energy gap begins due to this sharp increase in the absorption coefficient occurs. Absorption coefficient increases with photon energy within the range $1.25-1.55 \mathrm{eV}$ approximately (Fig. 6). Optical band gap calculated for the material was between 1.34 and $1.41 \mathrm{eV}$. Thus it can be said that the value of optical band gap of GaAs obtained was similar to those found by other workers (Sturge 1962, El-Nahass et al. 2011). For pure GaAs the value of band gap is usually found to be near $1.4 \mathrm{eV}$ (Omar 2003, BoylStad 2002), here present authors obtained the value a little lower than that which may be the 
effect of heavy doping (in the order of $10^{18} / \mathrm{cm}^{3}$ ). Usually band gap narrowing occurs in GaAs at higher impurity levels (Hudait et al. 1997, El-Nahass 2011) and it is directly proportional to the cubic root of carrier concentration.

\section{CONCLUSIONS}

From the present work, it can be summarized that, the carrier concentration of the p-type GaAs sample is in the order of $10^{18} / \mathrm{cm}^{3}$. So, the sample is heavily doped. Photoconductivity remains almost constant with the increase of sample current between 0.1 and $0.25 \mathrm{~mA}$. It increases nonlinearly with the increase of light intensity between 37 and 12780 lux. It increases almost linearly with the increase of temperature within the range $308-428 \mathrm{~K}$. The absorption coefficient increases parabolically with the frequency above the fundamental absorption edge of the curve and p-type material of GaAs has direct band gap. The material shows shrinkage in band gap due to heavy doping. It can be said that, the material may be suitable for fabrication of photoconductive devices.

\section{ACKNOWLEDGEMENT}

Authors are thankful to Experimental Physics Division, Atomic Energy Centre, Dhaka providing necessary facilities.

\section{REFRENCES}

Akhtar, N. and T. Begum. 2005. Electrical and Optical Properties of p-type Silicon in the temperature range 300 - 415 K. Nucl. Sci. \& Appl. 14(2): 34-38.

Akhtar, N., T. Begum, Z.Mozumder and A.K. Roy. 1999. A study of photoconductivity and carrier lifetime in p-type Germanium samples under different surface conditions. J. Bangladesh Academy of Science 23(1): 79-86.

Brehm, G. E. and G. L. Pearson. 1972. Gamma-radiation damage in epitaxial GaAs. J. Appl. Phys. 43: $568-574$.

BoylStad, R. L. and Louis Nashelsky. 2002. Electronic Devices and Circuit Theory. $8^{\text {th }}$ edition, Pearson Education, Inc., P-3-9.

Chowdhury, A.S., S. Choudhury, T. Begum and N. Akhter, 2010, Optical and transport properties of n-type GaAs, Dhaka Univ. J. Sci. 58(1): 109-112.

El-Nahass, M. M., S. B. Youssef, H. A. M. Ali. 2011. Optical properties of Zn doped GaAs single Crystals. J. Optoelectronics and Advanced Materials, 13(1): 82- 86.

Hudait, M.K., P. Modak, S. Hardikar, and S. B. Krupanidhi 1997. Zn incorporation and band gap shrinkage in p-type GaAs. J. Appl. Phys. 82: 10.

Joshi, G.P., R. Mangal, N.S.Saxena and T. P. Sharma. 2002. Band gaps of nanocomposites. Ind. J. Pure \& Appl. Phys. 297-300.

Knol, M. 1959. Material and Processes in Electron devices. 267.qa1. 
Longeaud, C., J. A. Schmidt, R. R. Koropecki. 2006. Determination of semiconductor band gap state parameters from photoconductivity measurements. II Experimental results. Phys. Rev. B 73: 235317.

M. Baublitz, Jr. and A.L Ruoff. 1982. Diffraction Studies of High Pressure Phases of GaAs and GaP. J. Appl. Phys. 53: 6179-6185.

Madelung, O. 1964. Physics of the III-V compounds. John Wiley, New York, 11, 68.

Mihailova, Ts. 2005. Structural defects in Gallium Arsenide. Journal of Optoelectronics and Advanced Materials, 7(1): 521-524.

Omar, M.A. 2003. Elementary Solid State Physics. Principles \& Application edition, Pearson Education Asia Pte Ltd. 259, 292, 295.

Ryvkin, S. M. 1964. Photoelectric Effect in Semiconductors. Consultant Bureau, New York, 2040.

Sadwick, L.P., P.P. Lee, M. Patel, M. Nikols, R.J. Hwu, J. E. Shield, D.C Streit, D. Brehmer, K. McCormick, S.J. Allen, R.W. Gedridge. 1996. Epitaxial dysprosium phosphide grown by gas source and solid-source MBE on GaAs substrates. J. Crys. Growth 164: 285-290.

Schroder, D.K. 1998. Semiconductor material and device characterization. John Wiley, Inc. New York, 596.

Sturge, D.M. 1962. Optical absorption of gallium arsenide between 0.6 and $2.75 \mathrm{eV}$. Phys. Rev. 127: 768-774.

(Received revised manuscript on 7 February, 2012) 